\title{
INDUSTRIAL BIG DATA ANALYTICS AND CYBER-PHYSICAL SYSTEMS FOR FUTURE MAINTENANCE \& SERVICE INNOVATION
}

\author{
Temitope O Awodiji \\ Computer Information Science Personnel, \\ California Miramar University, California, USA.
}

\begin{abstract}
Based on Information and Communication Technologies (ICT) fast advancement and the integration of advanced analytics into manufacturing, products, and services, several industries face new opportunities and at the identical time challenges of maintaining their ability and market desires. Such integration, that is termed Cyber-physical Systems (CPS), is remodeling the industry into a future level. CPS facilitates the systematic conversion of big data into information that reveals invisible patterns of deterioration and inefficiency and leads to better decision-making. This project focuses on existing trends within the development of industrial huge information analytics and CPS. Then it, in brief, discusses a system architecture for applying CPS in manufacturing referred to as 5C. The 5C architecture, comprises necessary steps to totally integrate cyber-physical systems within the manufacturing industry.
\end{abstract}

\section{KEYWORDS}

Information and Communication Technologies (ICT), Big Data, Analytic, Data, Data Science, Data Architecture, Cyber Physical Systems, Integration.

\section{INTRODUCTION}

Unmet needs in today's business During the last decade's manufacturers and service providers have taken an important step to improve the standard of products and services and to optimize their processes to survive in the competition and to react to market demands. Customer orientation, value creation, and service orientation quality oriented This development have led to the development of Prognostics and Management (PHM). Information and knowledge about the invisible patterns of asset depreciation. and the inconsistencies and inefficiencies of the processes. These patterns are invisible until an error occurs [(Lee, Lapira, Bagheri, Kao, (2013)] posits that the invention of such underlying patterns avoids the expensive failures and unplanned downtime of machinery. Such a maintenance scheme results in larger quality sustainability nearzero breakdown.

\section{CPS 5C LEVEL ARChiteCture}

In recent years, the rapid advancement of information and communication technologies (ICT) has accelerated the use of advanced sensors, data collection devices, wireless communication devices, and remote computing solutions. The integration of advanced analytics with communication technologies in close connection with physical machines is known as CyberPhysical Systems (CPS). According to Shi, Wan, Yan, and Suo (2011), "An Overview of Cyber- 
Physical Systems". Since the inception of its concept, CPS has been an ever-growing terminology in today's evolving industry. Predicting Potential Failures By implementing this predictive analysis in addition to a support system of your choice, the right services can be requested, and action can be taken to maximize the uptime, productivity, and efficiency of industrial systems. As the central hub for data management at the fleet level, CPS plays a crucial role in achieving the goals mentioned above.

\section{INTERNET OF THINGS AND EVOLUTION OF INDUSTRIES}

According to The Internet of Things (IoT), Kopetz [(2011)] posits that the internet of things. In real-time systems will gather, sort, synchronize and organize the data from totally different sources within a manufacturing plant or business. It provides a seamless, connected data management platform with real-time streaming and processing capabilities. This platform offers the possibility to implement a predictive analysis of big data for the transformation of data into information, knowledge, and actions through a CPS structure. Data to Action has the potential to add value to different parts of a business chain. For example, valuable data on hidden deterioration or inefficiency patterns between machines or production processes can lead to intelligent and effective maintenance options that avoid costly downtime and unplanned downtime.

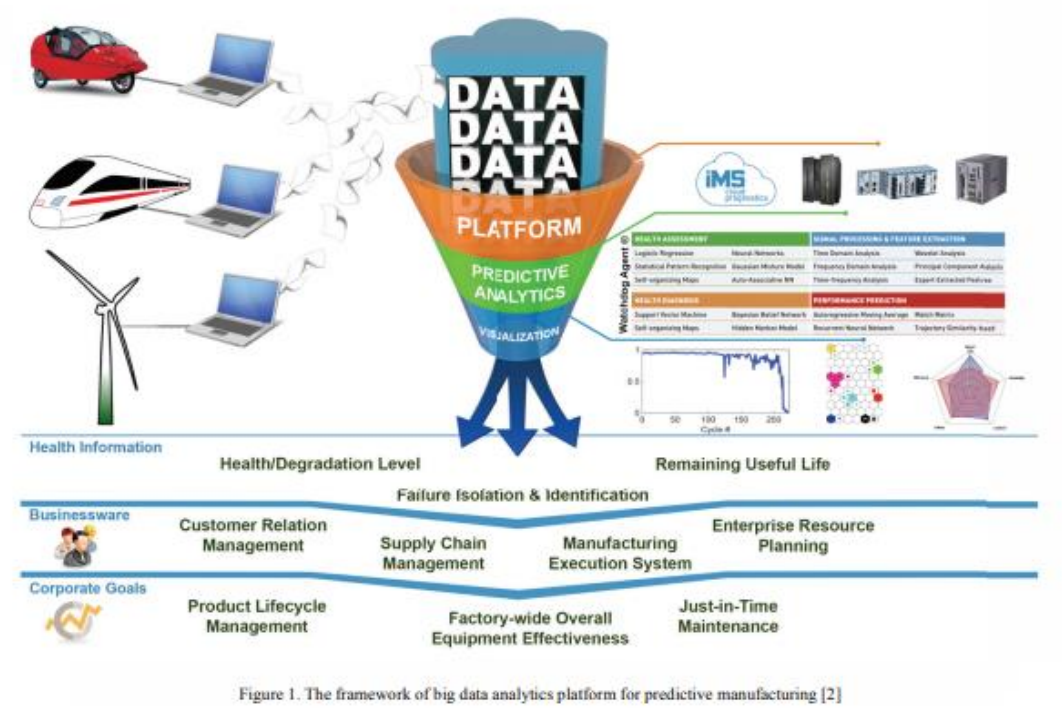

Figure 1 provides a schematic view of how massive data analytics will produce value among completely different sections of industries. Within the next section, the structure of CPS alongside its implementation aspects is mentioned.

\section{Cyber-Physical Systems}

The CPS structure, projected in Lee, J., B., Bagheri, H. A., Kao (2013), consists of five levels specifically $5 \mathrm{C}$ architecture. This framework provides a guide for the development of CPS for industrial applications. This CPS structure consists of 2 main components:

1) the advanced connectivity that ensures real-time information streamlining from the physical space to cyberspace and feedback from the cyberspace; and 
2) Intelligent data analytics that constructs the cyberspace. The Projected 5C framework provides advances that show how a CPS system can be built from data acquisition to value creation. The framework of CPS at completely different levels is shown in Figure 2. The 5C structure consists of good affiliation, Data-to-info Conversion, Cyber, cognition and Configuration levels.

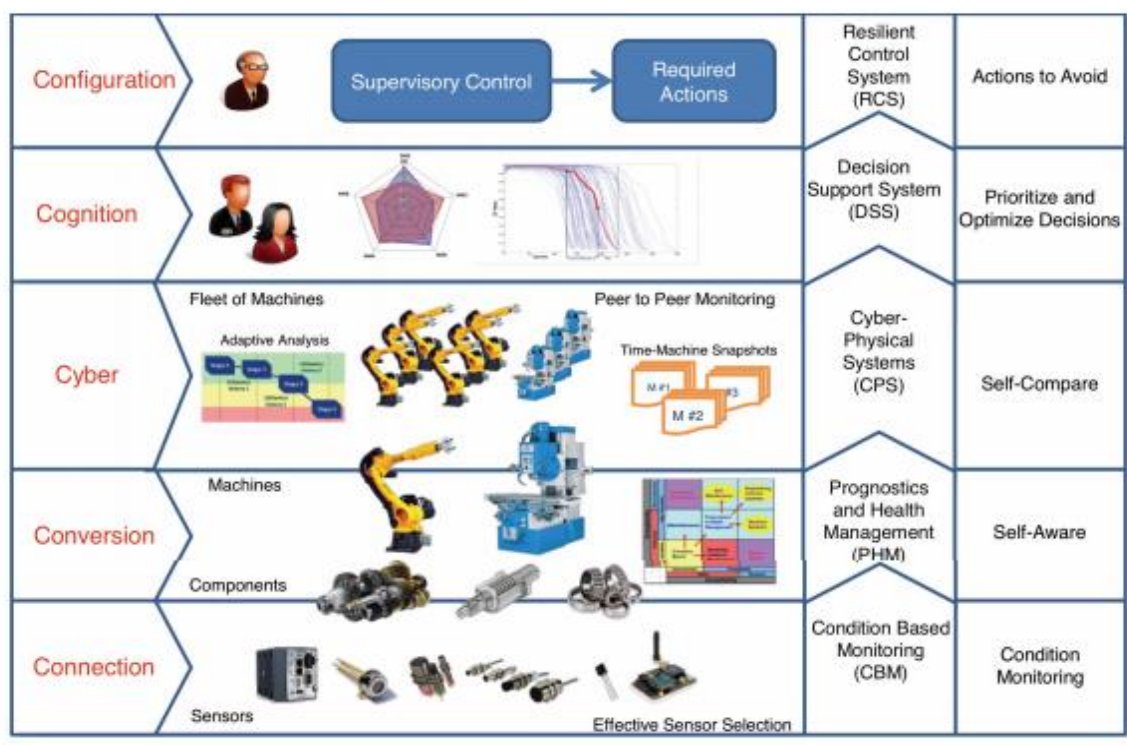

Figure 2. Framework of a cyber-physical system in different levels from data to action [5]

\subsection{Smart Connection}

This level consists of seamless and seamless strategies for managing data acquisition systems, optimizing data, and transferring it to the central server, selecting the right sensors, data sources and transfer protocols such as MT Connect. [Vijaya Raghavan, Sobel, Fox, Dornfeld and Wendorff (2008)]during this stage will have a great impact on the performance of the CPS within the following stages and on the quality and precision of the knowledge obtained by the system.

\subsection{Data-To-Information Conversion}

The core of such architecture is where the information is analyzed and remodeled into valuable data. Recently, there has been an in-depth focus on developing intelligent algorithms and data processing techniques. Such algorithms may be applied to numerous data sources, from machinery and process information to business and enterprise management data.

\subsection{Cyber}

The cyber level acts as the central information center in this architecture. Information is being pushed to that from each source and compiled to ascertain cyberspace. Having huge data gathered, specific analytics should be used to extract additional data that gives better insight into the status of individual machines among the fleet. 


\subsection{Cognition Implementing}

Cognition Implementing CPS upon this level generates a thorough information of the monitored system. correct presentation of the acquired knowledge to professional users supports the right decision to be taken.

\subsection{Configuration}

The configuration level is the feedback from cyberspace to the physical area and acts as a supervisory controller to create self-configuring and self-adapting machines. This stage acts as a resilience control system (RCS) to use the corrective and preventive choices, that has been created within the knowledge level, to the monitored system.

Cyber-physical System-based smart Machine Machining processes within the manufacturing business represent a highly dynamic and complex scenario for condition-based maintenance (CBM) and PHM. A CNC machine will sometimes handle a good vary of materials with completely different hardness and geometric shapes and consequently needs different combos of machine and cutting parameters to operate. traditional PHM methods are sometimes developed for a restricted range of machine varieties operating and dealing conditions and so cannot be used to effectively handle a complete manufacturing floor where machines may be utilized under a good range of working regimes that cannot be sculptured comprehensively beforehand. As a result, a CPS framework with the projected $5 \mathrm{C}$ structure is developed for sawing processes. The developed $\mathrm{Hz}$ for machine tools may be used to process and analyses machining information, evaluate the health condition of important parts (e.g., tool cutter) and further improves the instrumentation efficiency and dependability by predicting forthcoming failures, scheduling maintenance beforehand and adaptive management.

In factories, manufacturing processes begin with sawing massive items of material into selected sizes. because of the upstream nature of the sawing method, the quality and speed of sowing influence the complete production and any error may be propagated to the subsequent steps and end in dangerous quality product. As shown in figure 3, within the connectivity level, data is acquired from machines through each add-on sensors and controller signals. additionally, to the add-on vibration, acoustic emission, temperature, and current sensors, twenty control variables like blade speed, cutting time and blade height are force out of the PLC controller to provide a transparent understanding of the working status of every machine. the information is currently processed within the industrial computer connected to every machine.

At the conversion level, the industrial computer also performs the feature extraction and the data preparation. The feature extraction consists of extracting standard time-domain and frequency domain options such as RMS, kurtosis, waveband energy percentage, etc. from vibration and acoustic signals The calculated functions are sent together with the machine status data via the Wi-Fi network to the cloud server, where the function values are managed and maintained within the database. At the cyber level, the cloud server uses an adaptive clustering technique.[Yang, Bagheri, Kao and Lee (2015)]posited to segment the blade performance history (from when a brand-new blade was installed to now) into discrete operating regimes supported the relative change of the options comparison to the conventional baseline and therefore the local noise distribution (figure 4). The adaptive clump technique compares the present values of the features with the baseline and historical operating regimes. It identifies the most appropriate cluster from the history to match with this operating condition. Although, if none is found, the algorithmic rule generates a replacement cluster as a brand-new operating regime and generate connected health models for that regime. Further, if a similar operating condition happens, the algorithmic 
rule has its signatures in memory and can mechanically cluster the new data into that specific operating regime [Yang, S., B., Bagheri, H. A., Kao, J., Lee (2015)].

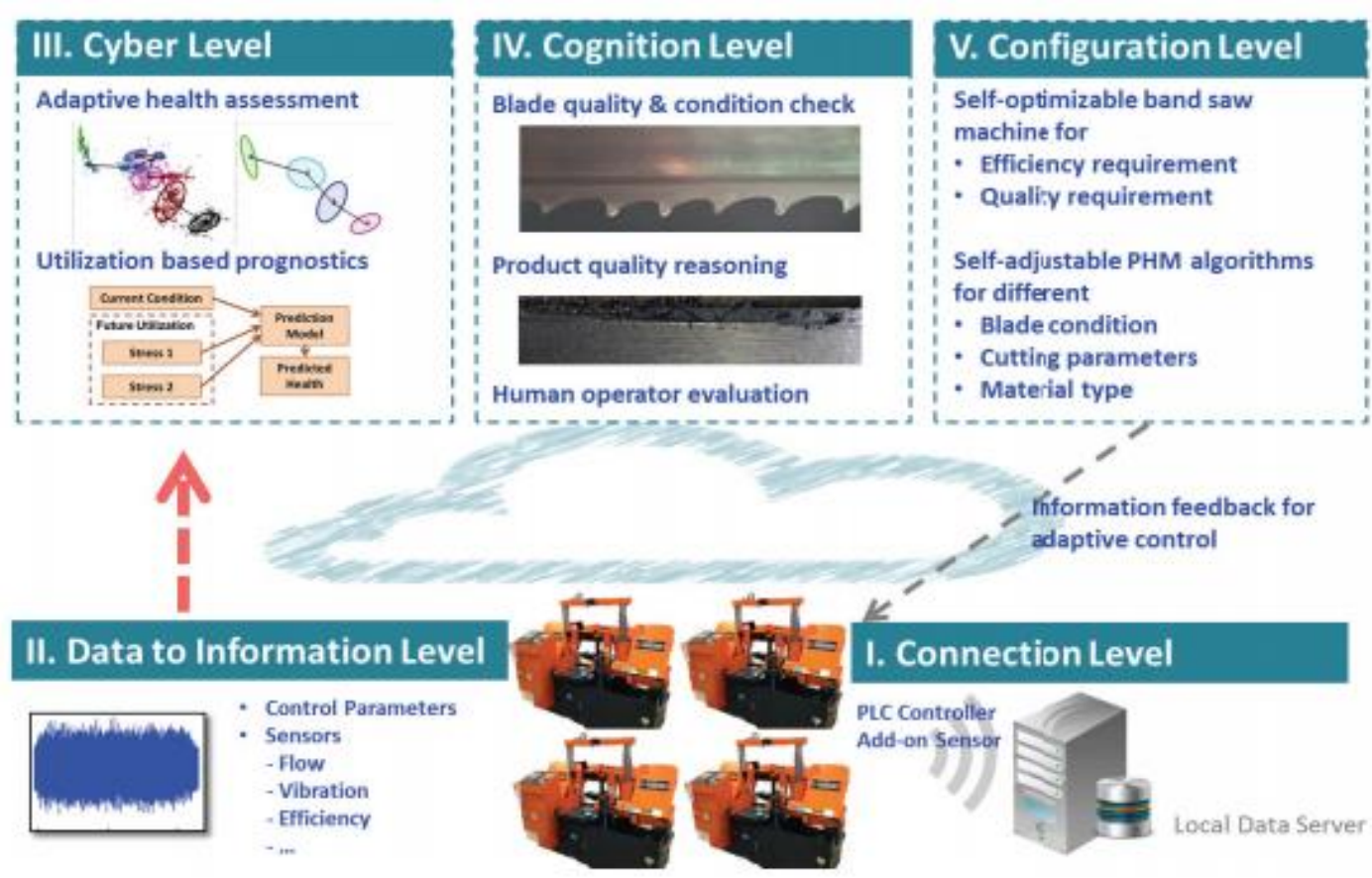

Figure 3. Overall Cyber Physical System setup for band saw Machines 

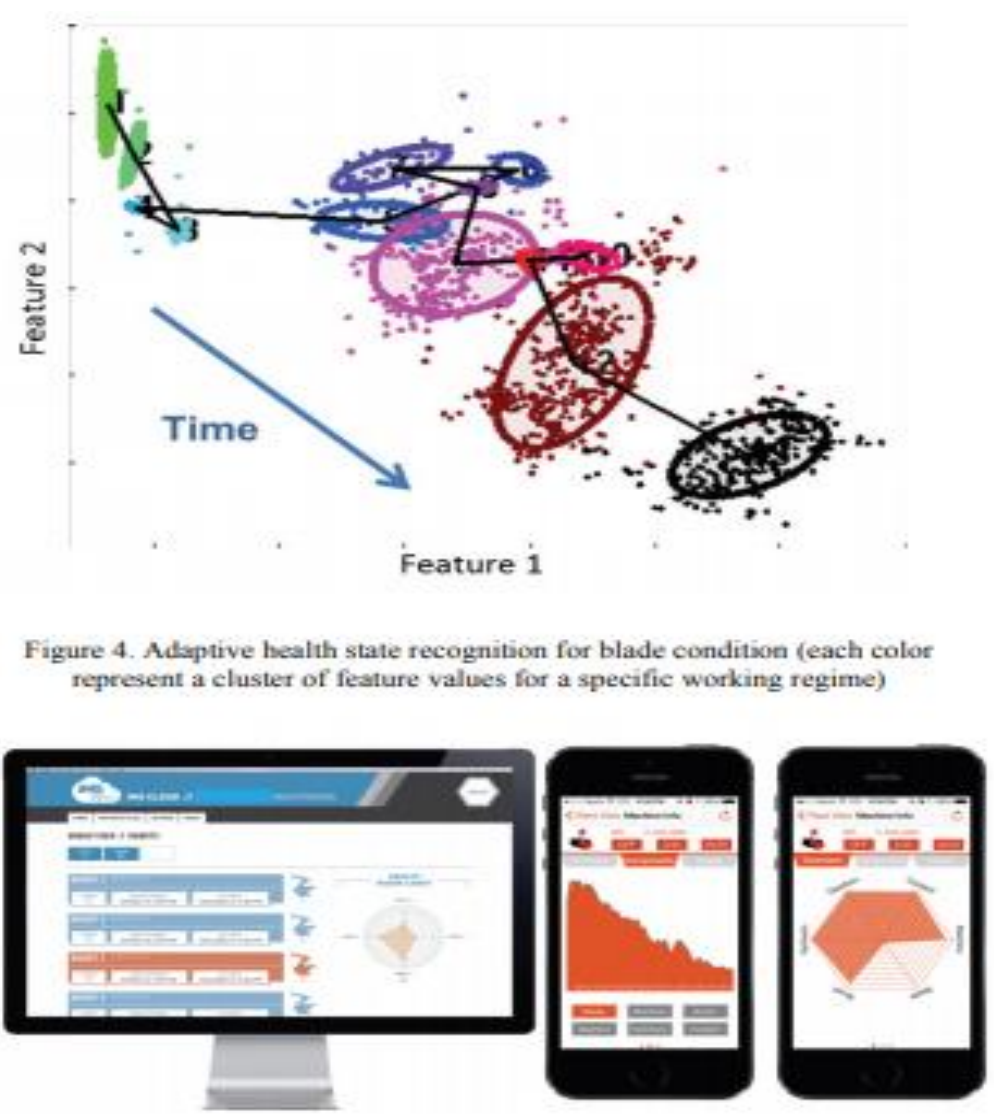

Figure 5. User interface for real-time access of machine health information

The health stages are further utilized in the cognition level and configuration level for improvement functions. for instance, once the blade is new, a better cutting speed is used for top productivity without hampering the standard, while after certain quantity of degradation has been detected, a a lot of moderate cutting ought to be applied to make sure quality. To help such decision-making process, web and iOS-based user interfaces are additionally developed so the health data of every connected machine tool is accessed in real time (figure 5).

\section{CONCLUSION}

This paper explained current trends toward implementing cyber-physical systems within the manufacturing industry. As managing industrial massive data has become a difficult task for factories, coming up with a generic architecture for implementing CPS in manufacturing is important. The $5 \mathrm{C}$ architecture that is mentioned in this article will change and centralize data processing, health assessment, and prognostics. This architecture covers all necessary steps, from data acquisition and processing of information to presentation for the user and decision-making support.

However, the health data generated by the system are used for higher-level functions such as maintenance planning and optimized control for higher productivity and overall system reliability. The capabilities of the 5C architecture have shortly demonstrated through a case study of CNC saw machines. The case study shows the integration of the $5 \mathrm{C}$ architecture for process and managing a fleet of CNC sawing machines that are normally utilized in manufacturing. the present integration of the 5C CPS architecture is in its early stage, therefore, advancement in all 
five levels of the architecture is practical. The cyber level contains high development potential through the development of new algorithms for the fleet-related analysis of machine performance in distributed data management systems.

\section{REFERENCES}

[1] Chan, F. T. S., H. C. W. Lau, R. W. L. Ip, H.K. Chan, S. Kong. "Implementation of total productive maintenance: A case study.". International Journal of Production Economics. 2005, 95.1: 71-94.

[2] Lee, J., E., Lapira, B., Bagheri, H. A., Kao. Recent advances and trends in predictive manufacturing systems in big data environment". Manufacturing Letters, 2013, 1.1: 38-41.

[3] Shi, J., J., Wan, H., Yan, H. Suo. "A survey of cyber-physical systems." Wireless Communications and Signal Processing (WCSP), 2011 International Conference on. IEEE, 2011.

[4] Kopetz, H. Internet of things. In: Real-time systems (pp. 307-323). Springer US. 2011.

[5] Lee, J., B., Bagheri, H. A., Kao. "A cyber-physical systems architecture for industry 4.0-based manufacturing systems." Manufacturing Letters, 2015, 3: 18-23.

[6] Vijaya Raghavan, A., W., Sobel, A., Fox, D., Dornfeld, P., Warnhoff. "Improving machine tool interoperability using standardized interface protocols: MT Connect." In: Proceedings of the 2008 international symposium on flexible automation (ISFA), 2008, Atlanta, GA, USA.

[7] Yang, S., B., Bagheri, H. A., Kao, J., Lee. "A Unified Framework and Platform for Designing of Cloud-based Machine Health Monitoring and Manufacturing Systems.", Journal of Manufacturing Science and Engineering, 2015.

\section{AUTHOR}

My Name is Temitope Awodiji, and I work remotely as a Data Analyst. I hold a master's degree in Computer Information Science. I am an Efficient Data Analyst professional with expert skills in SQL, Power BI, Tableau, EXCEL, and other data analytics tools. My experience includes generating, manipulating, interpreting, and analyzing data in fast-paced delivery and operations. Growing up, I have always enjoyed solving puzzles. So, this is the same way I see Data Set. I see it as a puzzle I want to solve. Finding the patterns nobody sees is a challenge to me.

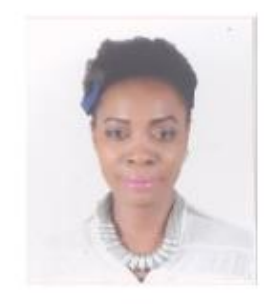

(C) 2021 By AIRCC Publishing Corporation. This article is published under the Creative Commons Attribution (CC BY) license. 